\title{
Enfermedad cerebrovascular en mujeres: estado del arte y visión del cardiólogo
}

\author{
Johanna P. Contreras ${ }^{a}$, Osmar Pérez ${ }^{b}$ y Natalia Figueroa ${ }^{c}$ \\ a Falla Cardiaca y Transplante Mount Sinai St. Luke's Hospital and Mount Sinai West, Icahn School of Medicine Mount Sinai, \\ New York, USA

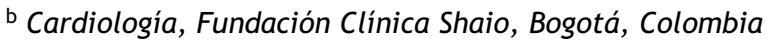 \\ c Facultad de Medicina, Universidad de los Andes, Bogotá, Colombia
}

Recibido el 20 de octubre de 2017; aceptado el 18 de diciembre de 2017

Disponible en Internet el 17 de enero de 2018

\section{PALABRAS CLAVE \\ Ataque \\ cerebrovascular; \\ Embarazo; \\ Fibrilación auricular}

\section{KEYWORDS}

Stroke;

Pregnancy;

Atrial fibrillation
Resumen La enfermedad cerebrovascular tiene varias denominaciones; en la actualidad se habla globalmente de ataque cerebrovascular, términos en los que se abarcan tanto manifestaciones hemorrágicas como isquémicas. Las mujeres son una población especial, con un riesgo relevante y mayores implicaciones pronósticas. Comparado con los hombres, comorbilidades como la hipertensión y la fibrilación auricular tienen mayor impacto vital y funcional en las mujeres. En el manejo no hay consideraciones que las diferencien de manera contundente, excepto en las mujeres embarazadas que requieren un ajuste especial al tratamiento, y en algunos casos no hay suficiente información para emitir una recomendación.

(c) 2017 Publicado por Elsevier España, S.L.U. en nombre de Sociedad Colombiana de Cardiología y Cirugía Cardiovascular. Este es un artículo Open Access bajo la licencia CC BY-NC-ND (http:// creativecommons.org/licenses/by-nc-nd/4.0/).

\section{Cerebrovascular disease in women: state of the art and a cardiologist's perspective}

\begin{abstract}
Cerebrovascular disease has several names and it is currently referred to as stroke, which encompasses hemorrhagic and ischemic manifestations. Women are a special population, with a relevant risk and greater prognostic implications. Comorbidities such as hypertension and atrial fibrillation have a greater impact at a vital and functional level, compared to men. Regarding management, there are no considerations that differentiate them in a definitive way, except in pregnant women (who require a special adjustment to the treatment), and in some cases there is not enough data to issue a recommendation.

(c) 2017 Published by Elsevier España, S.L.U. on behalf of Sociedad Colombiana de Cardiología y Cirugía Cardiovascular. This is an open access article under the CC BY-NC-ND license (http:// creativecommons.org/licenses/by-nc-nd/4.0/).
\end{abstract}




\section{Introducción}

La enfermedad cerebrovascular tiene múltiples denominaciones en la literatura, pero hoy se conoce como "ataque" o "accidente cerebrovascular"' (ACV). Recientemente, el Stroke Network avaló el uso del término "ataque cerebrovascular', el cual puede ser secundario a isquemia o hemorragia intracerebral y se define como una disfunción cerebral aguda con desarrollo de signos y síntomas focales o globales, que duran más allá de 24 horas y pueden acarrear la muerte $\mathrm{l}^{1,2}$.

Esta enfermedad es la segunda causa de muerte en el mundo, y es de gran importancia en el ámbito de la salud pública debido al gran impacto que tiene sobre la funcionalidad y el grado de dependencia que genera en los pacientes $^{3-5}$.

Las mujeres representan un grupo especial, dado que existen múltiples factores que las predisponen a mayor riesgo de desarrollar un ACV. Entre estos se han descrito diferencias en los factores de coagulación, factores hormonales, genéticos, inmunes y también sociales que finalmente contribuyen a este riesgo aumentado ${ }^{6}$. Adicionalmente, la fibrilación auricular es una importante causa de ACV en este género, la cual se incluye en los puntajes de riesgo para desarrollar ACV, sobre todo en mujeres mayores de 65 años $^{7}$.

\section{Epidemiología}

Varía según los diferentes grupos poblacionales; en la actualidad es la segunda causa de muerte y la principal causa de discapacidad en el mundo ${ }^{1,3}$.

Aproximadamente 16 millones de personas en todo el mundo se ven afectadas por un ACV cada año, y la prevalencia anual estimada de sobrevivientes después de este evento es de más de 60 millones $^{1,3,8}$.

Se ha descrito que la incidencia mundial de ACV isquémico es del $68 \%$, mientras que la incidencia de ACV hemorrágico (hemorragia intracerebral y hemorragia subaracnoidea combinada) es de $32 \%$. En Estados Unidos, la proporción de todos los ACV debido a isquemia, hemorragia intracerebral y hemorragia subaracnoidea es de 87, 10, y $3 \%$, respectivamente ${ }^{9}$.

La incidencia del ACV está disminuyendo en los países de altos ingresos, incluyendo los Estados Unidos; sin embargo está aumentando en los países de bajos ingresos, y en consecuencia el número de personas en el mundo con discapacidad y dependencia secundaria es alta y creciente ${ }^{10,11}$.

En los Estados Unidos, la incidencia anual de ACV nuevo o recurrente es de aproximadamente 795.000, de los cuales unos 610.000 se presentan por primera vez, y 185.000 son recurrentes ${ }^{10}$.

Los datos epidemiológicos en Latinoamérica son limitados secundario a la falta de estudios epidemiológicos que abarquen toda la población; no obstante, se ha calculado que la incidencia de enfermedad cerebrovascular en Latinoamérica oscial entre 0,89-1,83/1.000 personas $^{1,3}$.

En Colombia se han planteado diferentes estudios epidemiológicos para determinar la incidencia de esta enfermedad, entre los que está el estudio de prevalencia de factores de riesgo para enfermedad cerebrovascular isquémica en la población colombiana (FREC vi) ${ }^{12}$ y uno de los

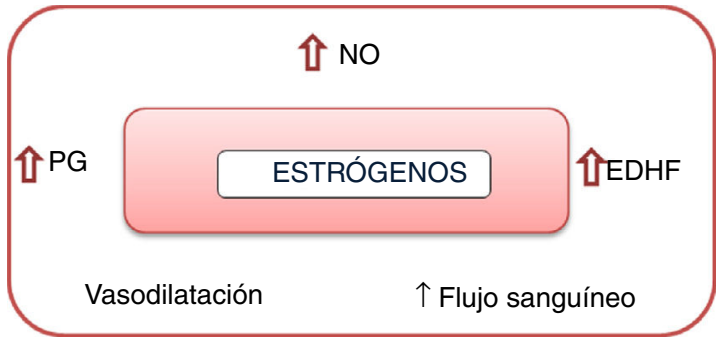

Figura 1 Mecanismos de acción de los estrógenos sobre la vasculatura. PG: prostaglandinas; NO: óxido nítrico; EDHF: factor de hiperpolarización derivado del endotelio ${ }^{16}$.

estudios más llamativos realizado en Sabaneta, Antioquia, el cual encontró una incidencia anual ajustada por edad y género de $0,89 / 1.000$ personas, siendo más común en hombres que en mujeres $(1,1 / 1.000$ vs. $0,61 / 1.000$ personas), cifra que se triplicó después de los 60 años ${ }^{1,3}$.

Respecto a la mortalidad, en Colombia se ha encontrado que es cercana al $15 \%$ a 30 días y se ha descrito que de cada 100 colombianos hospitalizados por un ACV, 37 tendrán algún grado de dependencia (Rankin < 3) y 24 necesitarán asistencia para sus actividades de la vida diaria a los 30 días $^{3}$.

El impacto sobre la calidad de vida, la productividad y el costo del cuidado de la salud es inmenso. Este se ha intentado evaluar con diferentes escalas en distintos estudios; sin embargo, no existe ninguna validada hasta ahora. Así mismo, se han descrito ciertos factores de riesgo que influyen en el pronóstico de estos pacientes, entre estos, la edad, la gravedad y el mecanismo del ACV, la localización, los hallazgos clínicos, las comorbilidades del paciente y las complicaciones relacionadas ${ }^{13,14}$.

En lo que respecta a los últimos años, se ha reportado una disminución de la incidencia de ACV en los estudios poblacionales de países desarrollados, la cual es más marcada en hombres que en mujeres (30,3 vs. $17,8 \%)^{14}$. No obstante, cabe resaltar que más mujeres son admitidas con ACV debido a la mayor expectativa de vida en este grupo ${ }^{15}$.

\section{Mecanismos biológicos: estrógenos y progesterona}

Las hormonas sexuales, incluidas la progesterona, los estrógenos y la testosterona, influyen en la fisiología vascular, la reactividad vascular, la barrera hematoencefálica y la aterosclerosis $^{14}$. Los vasos sanguíneos y en particular la vasculatura cerebral, son blanco de acción de estas hormonas debido a la expresión de receptores específicos para las hormonas sexuales ${ }^{16}$.

El estrógeno y la progesterona ejercen varios efectos sobre los vasos sanguíneos (fig. 1); entre estos está su efecto vasodilatador, que conlleva aumento en el flujo sanguíneo. Por otro lado, la testosterona tiene el efecto opuesto, pues lleva a la vasoconstricción de la vasculatura ${ }^{16}$.

Lo estrógenos mejoran la producción de óxido nítrico gracias al efecto sobre la óxido nítrico sintetasa endotelial. La reactividad vascular se ve afectada por aumento de la producción de óxido nítrico (NO), inducción de prostanoides vasodilatadores e influencia del factor de hiperpolarización derivado del endotelio (EDHF). Adicionalmente, al 
haber mayores niveles de NO circulante en las mujeres, hay mayores efectos sobre la barrera hematoencefálica y la autorregulación de la perfusión cerebral. En consecuencia, las arterias cerebrales masculinas tienden a tener un nivel más alto de vasoconstricción en respuesta a la presión arterial, comparado con las arterias cerebrales femeninas. Adicionalmente, el estrógeno mejora la sensibilidad de los vasos sanguíneos a sustancias vasodilatadoras e incluso puede llegar a contrarrestar los efectos vasoconstrictores de otras sustancias ${ }^{16}$.

El estrógeno y la progesterona reducen la formación de placas ateroscleróticas por sus efectos sobre las células del músculo liso, y es por esto que el primero ha sido considerado como posible tratamiento en la prevención de la enfermedad vascular en mujeres posmenopáusicas y se considera como agente protector en mujeres premenopaúsicas $^{17}$.

Sin embargo, existe cierta controversia respecto al factor protector que se le atribuye a los estrógenos, ya que algunos investigadores sugieren que los niveles circulantes de andrógenos en hombres pueden tener efectos adversos sobre la vasculatura cerebral y por lo tanto explicar el mayor riesgo que tiene esta población, pero esto es un campo que requiere mayor investigación ${ }^{16}$.

\section{Factores de riesgo específicos en mujeres}

Existen múltiples factores de riesgo para desarrollar un ACV, entre los que se han descrito algunos específicos del sexo femenino y otros que son más prevalentes en esta población (tabla 1$)^{6}$. Más adelante se discutirá de manera detallada desde el punto de vista de la cardiología, algunos de los principales factores de riesgo descritos en mujeres.

\section{Periodo de embarazo y posparto}

Dado que el riesgo de presentar eventos trombóticos aumenta durante el embarazo, se ha descrito una incidencia de ACV durante este período que oscila entre 9-34/100.000 partos en todo el mundo. Se postula que el aumento del riesgo de ACV durante el embarazo y el posparto, se debe a cambios hemostáticos que conducen a aumento de los factores de coagulación, disminución de factores anticoagulantes y factores fibrinolíticos. Adicionalmente, se ha descrito la resistencia a la proteína $\mathrm{C}$, los niveles más bajos de proteína $\mathrm{S}$ y el aumento del fibrinógeno, que combinados conducen a un estado hipercoagulable que aumenta el riesgo de $\mathrm{ACV}^{17,19}$.
Los trastornos hipertensivos del embarazo conllevan riesgo adicional para el desarrollo de ACV isquémico y hemorrágico, por esto es de gran importancia su tamizaje y diagnóstico oportuno en las mujeres embarazadas. Al respecto, las guías de la American Heart Association (AHA) recomiendan el uso de Aspirina a bajas dosis desde las 12 semanas de gestación hasta el final del embarazo (clase I, nivel de evidencia A), así como el suplemento de calcio diario en mujeres embarazadas que padecen hipertensión arterial ${ }^{6}$.

En cuanto al manejo antihipertensivo se recomienda el uso de medicamentos seguros y eficaces tales como metildopa, labetalol y nifedipino, teniendo en cuenta los efectos secundarios maternos y fetales (clase ।, nivel de evidencia A) ${ }^{6}$. Adicionalmente, se debe tener en cuenta que el atenolol, los bloqueadores de los receptores de angiotensina y los inhibidores directos de la renina están contraindicados durante el embarazo y no deben utilizarse por sus efectos teratogénicos ${ }^{6}$.

En lo concerniente a la vigilancia de las mujeres con hipertensión crónica, se recomienda continuar con sus regímenes antihipertensivos habituales con los ajustes de dosis y medicamentos necesarios, y adicionalmente ser monitorizadas de cerca por el riesgo de preeclampsia postparto ${ }^{6}$.

\section{Trombosis de los senos venosos en el embarazo}

La trombosis de los senos venosos (TSV) es otra de las presentaciones clínicas del ACV en mujeres y se describe con mayor frecuencia en el embarazo ${ }^{18}$. En una gran serie de pacientes con TSV el 75\% eran mujeres, de las cuales el $17 \%$ estaban embarazadas o en período posparto ${ }^{19}$.

La sintomatología en el embarazo no difiere de las mujeres no embarazadas, reportándose con mayor frecuencia la aparición de una cefalea aislada intensa ${ }^{19}$.

Una revisión sistemática reciente encontró que el riesgo de TSV y otros tromboembolismos venosos durante el embarazo aumentan después de una TSV previa, encontrándose una recurrencia hasta en $9 / 1000$ embarazos. Por consiguiente, el riesgo de TSV es lo suficientemente bajo para no aconsejar en contra de futuros embarazos en estas mujeres, pero se recomienda llevar un seguimiento cercano en caso de que se den, así como medidas para prevenir futuros eventos tromboembólicos ${ }^{20}$.

\section{La anticoncepción oral}

El consumo de anticonceptivos orales (ACO) en mujeres se ha asociado con un aumento del riesgo de ACV. Esto puede

Tabla 1 Factores de riesgo de ACV en mujeres ${ }^{6}$

Factores de riesgo específicos para el sexo femenino

- Embarazo

- Preeclampsia

- Diabetes gestacional

- Uso de anticonceptivos orales

- Uso de hormonas en la postmenopausia
Factores de riesgo más prevalentes en mujeres

- Cambios en el estado hormonal

- Migraña con aura

- Fibrilación auricular

- Diabetes mellitus

- Depresión

- Estrés psicosocial 
deberse a los efectos procoagulantes de los estrógenos y la progesterona, que conllevan aumento de la actividad de factores de coagulación como el fibrinógeno y a su vez, a la disminución de factores anticoagulantes ${ }^{21}$.

Un metaanálisis realizado por Gillum et $\mathrm{al}^{22}$., que analizó 16 diferentes estudios, encontró que las mujeres que consumían ACO tenían un riesgo relativo (RR) de 2,75 (intervalo de confianza -IC- 95\% de 2,24-3,38) para desarrollo de ACV 22 . Adicionalmente, este estudio encontró que aquellas mujeres que tomaban dosis bajas de estrógenos $(<0,5 \mu \mathrm{g})$ tenían RR de 2,08 (IC 95\% 1,55-2,8), menor comparado con aquellas que ingerían una dosis mayor (> 0,5 $\mu \mathrm{g}$ ) RR 4,53 (IC 95\% $2,17-9,5)^{22}$.

Existen varios factores de riesgo modificables en la población femenina, los cuales incrementan el riesgo de desarrollar un ACV con el consumo concomitante de ACO. Entre estos se encuentran el tabaquismo, la hipertensión arterial, la cefalea y la historia de tromboembolia pulmonar o de miembros inferiores. En el estudio de Ryan et $\mathrm{al}^{23}$., se encontró que las mujeres con más de un factor de riesgo de los mencionados, eran más susceptibles a un ACV con el uso de ACO, con un OR 3,12 (IC 95\% 1,62-6,00). Adicionalmente, encontraron que la combinación de factores más riesgosa era para aquellas mujeres con tabaquismo y cefalea ${ }^{23}$.

Sin embargo, el riesgo adicional de ACV que conlleva el consumo de anticonceptivos en la población femenina, no supera su beneficio en el manejo adecuado de la anticoncepción y los embarazos no deseados.

\section{Visión desde la Cardiología clínica del problema del ataque cerebrovascular en mujeres}

La enfermedad cerebrovascular debe ser una entidad que se aborde de manera integral, no solo por Neurología; por tanto, especialistas como cardiólogos e internistas, deben intervenir en el enfoque y manejo óptimo de esta entidad. Existen varios factores de riesgo, muchos ya mencionados previamente $^{6}$. Pero tal vez la principal preocupación y el motivo principal de interconsulta de Neurología para los cardiólogos es el contexto de un ACV de fuente indeterminada, y ante la alta sospecha de cardioembolia, como causa más prevalente del evento isquémico, se promueve una búsqueda eficaz y completa ${ }^{24}$.

Se reporta que la fibrilación auricular (FA) es la arritmia más común en el mundo, la cual se asocia con morbimortalidad, y es una causal de cardioembolia, produciendo los ACV descritos como más graves y discapacitantes si se comparan con otras etiologías de $A C V^{15,25}$.

El riesgo de desarrollar esta arritmia es 1,2-2,0 veces mayor en hombres, pero tiende a ser más prevalente en mujeres, dado que estas tienen una expectativa de vida más larga y representan una mayor proporción en la población ${ }^{25}$.

El riesgo atribuible de ACV por FA aumenta con la edad, y llega a ser hasta 4 a 5 veces más probable; por esto se calcula que habrá un número creciente de mujeres de edad avanzada con diagnóstico de $\mathrm{FA}^{26}$.

En la publicación "Get with the guidelines stroke", un tercio de los ingresos hospitalarios por ACV fueron pacientes $\geq 80$ años de edad, entre los que identificó FA en el 15,6 y $20,4 \%$ de los hombres y las mujeres, respectivamente ( $p$ $<0,0001)^{27}$.
Las mujeres con FA reciben en menor proporción tratamiento anticoagulante en comparación con los hombres $(88 \%$ contra 89,7\%, OR ajustado, 0,93, IC del 95\%, 0,88-0,98) por razones que no son claras hoy en día ${ }^{28}$.

Para definir quienes se benefician de manera objetiva del tratamiento anticoagulante, se han elaborado herramientas de estratificación del riesgo, como el puntaje $\mathrm{CHA}_{2} \mathrm{DS}_{2}-\mathrm{VASc}$, que es útil para guiar la toma de decisiones para la instauración de terapia anticoagulante y la prevención de ACV isquémico cardioembólico ${ }^{29}$.

En esta escala se tiene en cuenta la historia de falla cardiaca (1 punto), hipertensión arterial (1 punto), edad entre 65 y 74 años (1 punto), 75 años o más (mayor riesgo, 2 puntos), antecedente de ACV previo (2 puntos), enfermedad vascular previa (1 punto) y sexo femenino (1 punto si se adiciona a otro factor de riesgo) $)^{7,29}$.

La clasificación de $\mathrm{CHA}_{2} \mathrm{DS}_{2}$-VASc se recomienda ampliamente incluso por la Sociedad Europea de Cardiología y por la AHA para la clasificación de riesgo de $\mathrm{ACV}^{30,31}$.

El sexo femenino es, por tanto, un predictor independiente del ACV en pacientes con FA y se ha comprobado con diferentes estudios, con grandes cohortes de pacientes la interacción edad-sexo en aquellos con FA, encontrándose mayor riesgo de ataque cerebrovascular en mujeres de 75 años o más, en comparación con los hombres ${ }^{26,32}$.

En guías actuales, tanto americanas como europeas, se coincide en anticoagular con un puntaje de $\mathrm{CHA}_{2} \mathrm{DS}_{2}$-VASc mayor o igual a 2 con indicación $\mathrm{IA}^{30}$. Sin embargo, los últimos consensos dejan la posibilidad abierta para anticoagular a criterio clínico aquellos pacientes con $\mathrm{CHA}_{2} \mathrm{DS}_{2}$-VASc de 1 en hombres y 2 en mujeres ${ }^{31}$.

Sin embargo, se deben tener en cuenta varias consideraciones. Primero, no todos los factores de riesgo en el $\mathrm{CHA}_{2} \mathrm{DS}_{2}$-VASc tienen igual peso, y la tendencia probablemente deba ser plantear anticoagulación oral para pacientes con fibrilación auricular y al menos un factor de riesgo para $\mathrm{ACV}$, balanceando el riesgo de sangrado y decidiéndolo en conjunto con el paciente ${ }^{31}$.

Segundo, se debe tener en cuenta que de acuerdo con el puntaje, el riesgo anual se incrementa; por ejemplo, se describe que con $\mathrm{CHA}_{2} \mathrm{DS}_{2}$-VASc de 1 el riesgo es de $1,3 \%$, y el máximo puntaje que es 9 da un riesgo de $15,2 \%{ }^{29}$.

Por tanto, es importante considerar la anticoagulación con puntaje de 1 , pues el riesgo no equivale a cero y hay estudios con gran cantidad de pacientes en los que se ha descrito un riesgo aumentado de $\mathrm{ACV}$ incluso en aquellos con $\mathrm{CHA}_{2} \mathrm{DS}_{2}-\mathrm{VASC}=1$ (y eran de sexo masculino), de ahí a que las más recientes guías europeas, pongan a consideración anticoagular estos pacientes ${ }^{7}$.

Sin embargo, conviene aclarar que si el único factor de riesgo es ser mujer, para poder sumar esta condición se debe tener al menos un factor de riesgo adicional, como edad mayor o igual a 65 años. En las guías europeas más recientes, si se es mujer se tiene un factor de riesgo adicional (puntuaría 2) y se plantearía anticoagulación, pero si solo se es mujer sin otro factor de riesgo puntuaría 1 y no se anticoagularía ni se antiagregaría (la antiagregación sola no previene eventos en pacientes con FA y sí aumenta el riesgo de sangrado). Adicionalmente, en los hombres que puntúen 1 , se da la opción de anticoagular (recomendación IlaB) ${ }^{31}$.

Respecto al diagnóstico, cardiólogos y neurólogos deben estar encaminados a descartar etiología embólica, y como 
ya se mencionó previamente la fibrilación auricular es una causa líder y se debe precisar su diagnóstico mediante diferentes métodos, inicialmente electrocardiograma (ECG); es común que se inicie con la solicitud de un Holter electrocardiográfico. Sin embargo, el rendimiento diagnóstico del Holter electrocardiograma (ECG) de 24 horas, suele ser bajo para diagnosticar FA y depende de la frecuencia en que se presenten los síntomas (palpitaciones principalmente) ${ }^{33}$.

Es por esto que cada vez hay mayor evidencia de la monitorización de eventos con registros electrocardiográficos más extensos que van desde semanas a meses con monitores externos o implantables, y se ha encontrado que aquellos ACV criptogénicos o de fuente indeterminada son explicados por una FA no diagnosticada por métodos convencionales ${ }^{34,35}$.

Por otro lado, las modalidades diagnósticas imagenológicas como el ecocardiograma, aportan al diagnóstico y abordaje integral en el estudio etiológico del ACV, para descartar trombos intracavitarios, evaluar la función ventricular y el tamaño de las aurículas, así como tamizar defectos septales. De acuerdo con las necesidades diagnósticas y las características del paciente se recomendará abordaje transtorácico o transesofágico. En este contexto cada vez se describen más pacientes con tamaño aumentado de la aurícula izquierda (volumen $>34 \mathrm{ml} / \mathrm{m}^{2}$, o $>40 \mathrm{~mm}$ en $2 \mathrm{D}$ ) y su relación con ACV recurrente o de causa indeterminada, y a su vez este hallazgo se relaciona con aparición de fibrilación auricular e incapacidad de mantener ritmo sinusal con antiarrítmicos, y por consiguiente mayor riesgo de $\mathrm{ACV}^{36,37}$.

Otras causas menos frecuentes son el foramen oval persistente del cual hay controversia entre cerrar y no cerrar el defecto. En caso de no haber encontrado otra causa de $\mathrm{ACV}$ y en pacientes con historia de ACV o ataque isquémico cerebral transitorio (AIT) previo, podría indicarse el cierre percutáneo del defecto; sin embargo es una recomendación que no es fuerte y no es la causa más frecuente en mujeres, pero se ha descrito incluso en el embarazo ${ }^{38-40}$.

En cuanto al tratamiento y la prevención de ACV tanto por guías y sobre todo por la evidencia actual, los anticoagulantes directos (DOAC)- rivaroxabán, apixabán, dabigatrán y edoxabán- estarían indicados con un nivel de evidencia y recomendación $\mathrm{IA}$, al igual que los antagonistas de la vitamina $\mathrm{K}$ (AVK); no obstante, se recomienda el uso de los primeros, por encima de los segundos, dado su menor riesgo de sangrado, y por tanto mejor balance clínico neto, así como menor interacción con medicamentos o alimentos y adicionalmente seguridad sin necesidad de monitorización como sí es imprescindible en el caso de los $\mathrm{AVK}^{31,41}$.

Otras comorbilidades y/o factores de riesgo para ACV en mujeres en las que los cardiólogos deben intervenir, es en el control adecuado de la hipertensión arterial y de la dislipidemia ${ }^{6}$.

El control óptimo de la presión arterial en pacientes con ACV incluye el uso de inhibidores de la enzima convertidora de angiotensina (IECA) o antagonistas del receptor de angiotensina ॥ (ARA ॥) en combinación con calcio-antagonistas y/o diuréticos tiazídicos, de acuerdo con las cifras de presión arterial y metas que se quieran lograr con base en la edad (menos de 140/90 mm Hg y en mayores de 60 años menos de $150 / 90 \mathrm{~mm} \mathrm{Hg}^{6,42}$.

Los pacientes con enfermedad cerebrocardiovascular deberán recibir estatinas de alta intensidad (atorvastatina
40-80 mg día y rosuvastatina 20 - 40 mg día), y buscar mantener un colesterol de baja densidad (LDL), idealmente menor a $70 \mathrm{mg} / \mathrm{dl}$ en prevención secundaria, así como promover estilos de vida saludable, dieta balanceada, prevención y tratamiento de la obesidad ${ }^{6,43}$.

\section{Severidad del ataque cerebrovascular en mujeres y hallazgos neurológicos particulares}

Las mujeres se manifiestan clínicamente con ataques cerebrovasculares más graves que los hombres. Se estima que la tasa de pacientes que presentan un puntaje del National institute of Health Stroke Scale (NIHSS) > 7 (por tanto mayor severidad), corresponde en un $44 \%$ de mujeres y a un $36 \%$ de hombres $^{15}$.

Las mujeres con mayor frecuencia presentan síntomas inusuales, y con menor periodicidad se describen síntomas como disartria, ataxia y parestesia. Por ejemplo, en las mujeres se observa mayor pérdida de sueño e inconsciencia $(26,7 \%)$ que en hombres $(19,7 \%)$, basándose en los datos del International Stroke Trial ${ }^{44}$.

En resumen, las mujeres pueden tener un perfil ligeramente diferente en la presentación clínica que los hombres, hecho que infortunadamente causa un diagnóstico tardío y a su vez, puede conducir a la demora de su tratamiento, reduciendo así la oportunidad de un pronóstico favorable ${ }^{45}$.

En consecuencia, esto se traduce en una estancia hospitalaria más larga y morbilidad y mortalidad elevadas. Por ende, es fundamental emprender más investigaciones y estudios para abordar esta problemática y alertar a los médicos clínicos a estar más alerta a identificar banderas rojas y signos de alarma tempranos ${ }^{46}$.

\section{Particularidades del tratamiento en mujeres}

En la actualidad, el tratamiento del ACV en hombres y mujeres se hace con base en guías y no diferencia un sexo del otro, a menos que se trate de contextos como el embarazo o de estrategias preventivas, como se mencionó, cuando hay relación FA y $\mathrm{ACV}^{2}$.

Sin embargo, en cuanto a la evidencia aportada por la literatura, las mujeres están generalmente subrepresentadas en los estudios clínicos aleatorizados y esto limita la extrapolarización de los hallazgos a esta población. En la mayoría de los estudios los hombres representan al menos dos tercios de la muestra y la edad media permanece por debajo de los 70 años ${ }^{25}$.

Una de las terapias de mayor impacto tanto en sobrevida como en pronóstico funcional evaluada por la escala de RANKIN, es una trombólisis oportuna en el tiempo de ventana establecido por guías, es decir 4,5 horas para trombólisis endovenosa y hasta 6 horas para manejo intraarterial y endovascular ${ }^{47-50}$. En cuanto a la terapia trombolítica, no hay indicaciones de diferencias de género en cuanto a beneficio y riesgo ${ }^{51}$.

Respecto al tratamiento endovascular, un metaanálisis de cinco ensayos no encontró diferencias de género acerca del efecto del tratamiento ajustado en la escala de Rankin modificada a los 90 días. En lo concerniente a esta terapia se ha visto una tendencia a una menor estancia hospitalaria en hombres comparado con mujeres, lo cual puede deberse a 
que los hombres tienden a presentar esta condición a una edad menor que las mujeres y usualmente con cuadros clínicos menos severos ${ }^{47}$

En las intervenciones de arteria carótida, se han reportado diferencias significativas de género. El sexo femenino se considera un riesgo quirúrgico con endarterectomía, que conduce a una frecuencia más alta de ACV y muerte perioperatoria.

El manejo endovascular con stents carotídeos también parece tener una incidencia más alta de eventos adversos en mujeres ${ }^{52}$.

En cuanto a fibrilación auricular, un metaanálisis sobre las diferencias de género, evaluó la seguridad y eficacia de antagonistas de la vitamina K (AVK) y anticoagulantes orales directos (DOAC), reportándose una tendencia hacia una mayor protección contra la tromboembolia en mujeres junto con un menor riesgo de hemorragia con la administración de DOAC; sin embargo, el tiempo en rango terapéutico (TRT) para AVK no se incluyó en el análisis, lo cual deja cierta incertidumbre sobre la influencia en los resultados ${ }^{53}$.

Hay varias consideraciones en cuanto a las particularidades que pueden influir en la respuesta a las diferentes terapias del ACV en mujeres, y se requieren más estudios para esclarecerlo; sin embargo se han descrito algunas como por ejemplo: mayor edad de presentación, que hace más probables las complicaciones, mayor número de comorbilidades y mayor probabilidad de polifarmacia (más interacciones farmacológicas) ${ }^{54}$.

\section{Conclusiones}

La enfermedad cerebrovascular en la mujer tiene varias particularidades, que van desde su etiología hasta su pronóstico. Los factores hormonales propios del sexo, el uso de anticonceptivos orales y situaciones como el embarazo influyen de manera importante en su presentación. Infortunadamente en las mujeres, el ACV se presenta a edades más avanzadas lo cual se asocia con peor pronóstico funcional y vital. En condiciones especiales como el embarazo se debe hacer control óptimo de los factores de riesgo debido a la ausencia de terapias eficaces con evidencia concreta.

La enfermedad cerebrovascular requiere manejo multidisciplinario para lograr la prevención y el control de los factores de riesgo, como también en el tratamiento de comorbilidades como la fibrilación auricular, la hipertensión arterial, las dislipidemias y la obesidad.

Es clave que en los próximos años se desarrollen estudios que incluyan específicamente una muestra significativa de mujeres, que permitan entender mejor la etiología, el diagnóstico y el tratamiento del ACV en mujeres.

\section{Conflicto de intereses}

Ninguno.

\section{Bibliografía}

1. Silva FA, Zarruk JG, Quintero C, Arenas W, Silva SY. Enfermedad cerebrovascular en Colombia. 2006;13:85-9.

2. Jauch EC, Saver JL, Adams HP, Bruno A, Connors JJB, Demaerschalk BM, et al. Guidelines for the early management of patients with acute ischemic stroke: a guideline for healthcare professionals from the American Heart Association/American Stroke Association. Stroke. 2013;44:870-947.

3. Muñoz Collazos M. Cerebrovascular disease. Acta Neurológica Colomb. 26(2):59-61.

4. Latin American Stroke Registry (LARS) Colombian Branch: one year report. Cerebrovasc Dis. 2010; 29(suppl2): 217. [Internet]. [Acceso 13 Jan 2015]. Disponible en: http: //www.scielo.org.co / scielo. php?script=sci_nlinks\&ref $=000025 \&$ pid $=$ S0120$8748201000020000200001 \& \operatorname{lng}=e n$.

5. Guzik A, Bushnell C. Stroke epidemiology and risk factor management. 2017:15-39.

6. Bushnell C, McCullough LD, Awad IA, Chireau MV, Fedder WN, Furie $\mathrm{KL}$, et al. Guidelines for the prevention of stroke in women: A statement for healthcare professionals from the American heart association/American stroke association. Stroke. 2014;45:1545-88.

7. Chao TF, Liu CJ, Wang KL, Lin YJ, Chang SL, Lo LW, et al. Should atrial fibrillation patients with 1 additional risk factor of the $\mathrm{CHA}_{2} \mathrm{DS}_{2}$-VASc Score (Beyond Sex) receive oral anticoagulation? J Am Coll Cardiol. 2015;65:635-42.

8. Grysiewicz RA, Thomas K, Pandey DK. Epidemiology of ischemic and hemorrhagic stroke: incidence, prevalence, mortality, and risk factors. Neurol Clin. 2008;26:871-95, vii.

9. Mukherjee D, Patil CG. Epidemiology and the global burden of stroke. World Neurosurg. 2011;76 6 Suppl:S85-90.

10. Go AS, Mozaffarian D, Roger VL, Benjamin EJ, Berry JD, Blaha MJ, et al. Heart disease and stroke statistics-2014 update: a report from the American Heart Association. Circulation. 2014;129:e28-92.

11. Lozano R, Naghavi M, Foreman K, Lim S, Shibuya K, Aboyans V, et al. Global and regional mortality from 235 causes of death for 20 age groups in 1990 and 2010: a systematic analysis for the Global Burden of Disease Study 2010. Lancet. 2012;380:2095-128.

12. Patricia Bonilla N, Oliveros H, Proaños J, Espinel B, Álvarez JC, Duran C, et al. Estudio de frecuencia de los factores de riesgo asociados al desarrollo de enfermedad cerebrovascular isquémica no embólica en un hospital de tercer nivel. Acta Neurológica Colomb. 2014;30:149-55.

13. Muir KW, Weir CJ, Murray GD, Povey C, Lees KR. Comparison of neurological scales and scoring systems for acute stroke prognosis. Stroke. 1996;27:1817-20.

14. New PW, Buchbinder R. Critical appraisal and review of the Rankin scale and its derivatives. Neuroepidemiology. 2006;26: 4-15.

15. Santalucia P, Pezzella FR, Sessa M, Monaco S, Torgano G, Anticoli $S$, et al. Sex differences in clinical presentation, severity and outcome of stroke: Results from a hospital-based registry. Eur J Intern Med. 2013;24:167-71.

16. Krause DN, Duckles SP, Pelligrino DA. Influence of sex steroid hormones on cerebrovascular function. J Appl Physiol. 2006;101:1252-61.

17. Tate J, Bushnell C. Pregnancy and stroke risk in women. Womens Health (Lond Engl). 2011;7:363-74.

18. Ferro JM, Canhão P. Cerebral Venous Sinus Thrombosis: Update on Diagnosis and Management. Curr Cardiol Rep. 2014; 16(9.).

19. Zuurbier SM, Middeldorp S, Stam J, Coutinho JM. Sex differences in cerebral venous thrombosis: A systematic analysis of a shift over time. Int J Stroke., 11. UK: London, England: SAGE PublicationsSage; 2016. p. 164-70.

20. Aguiar De Sousa D, Canhaõ P, Ferro JM. Safety of pregnancy after cerebral venous thrombosis: a systematic review. Stroke. 2016;47:713-8.

21. Samai AA, Martin-Schild S. Sex differences in predictors of ischemic stroke: Current perspectives. Vasc Health Risk Manag. 2015;11:427-36. 
22. Gillum LA, Mamidipudi SK, Johnston SC. Ischemic stroke risk with oral contraceptives: a meta-analysis. JAMA J Am Med Assoc. 2000;284:72-8.

23. Kang P, Liao M, Wester MR, Leeder JS, Pearce RE. NIH Public Access. Ratio. 2010;36:490-9.

24. Hart RG, Diener HC, Coutts SB, Easton JD, Granger CB, O'Donnell MJ, et al. Embolic strokes of undetermined source: The case for a new clinical construct. Lancet Neurol. 2014;13:429-38.

25. Cha Y, Redfield MM, Shen W, Gersh BJ. Atrial fibrillation and ventricular dysfunction. Circulation. 2017:2839-43.

26. Friberg L, Benson L, Rosenqvist M, Lip GYH. Assessment of female sex as a risk factor in atrial fibrillation in Sweden: nationwide retrospective cohort study. BMJ. 2012;344:e3522.

27. Fonarow GC, Reeves MJ, Zhao X, Olson DM, Smith EE, Saver $\mathrm{JL}$, et al. Age-related differences in characteristics, performance measures, treatment trends, and outcomes in patients with ischemic stroke. Circulation. 2010;121:879-91.

28. Reeves MJ, Fonarow GC, Zhao X, Smith EE, Schwamm LH. Get With The Guidelines-Stroke Steering Committee \& Investigators Quality of Care in Women With Ischemic Stroke in the GWTG Program. Stroke. 2009;40:1127-33.

29. Lip GYH, Frison L, Halperin JL, Lane DA. Identifying patients at high risk for stroke despite anticoagulation: A comparison of contemporary stroke risk stratification schemes in an anticoagulated atrial fibrillation cohort. Stroke. 2010;41: 2731-8.

30. January CT, Wann LS, Alpert JS, Calkins H, Cleveland JC, Cigarroa E, et al. Guideline for the Management of Patients With Atrial Fibrillation. A Report of the American College of Cardiology. Circulation. 2014:1-124.

31. Task A, Members F, Kirchhof P, Uk C, Uk DK, Uk BC, et al., ESC Guidelines for the management of atrial fibrillation developed in collaboration with EACTS The Task Force for the management of atrial fibrillation of the European Society of Cardiology (ESC) 2016. Developed with the special contribution of the Europ. 2016:2893-962.

32. Humphries KH. Sex differences in stroke risk. 2012:3-9.

33. Mora-Pabón G. Evaluación de la fibrilación auricular mediante electrocardiograma y Holter. Rev Colomb Cardiol. 2016;23:27-33.

34. Gladstone DJ, Spring M, Dorian P, Panzov V, Thorpe KE, Hall J, et al. Atrial fibrillation in patients with cryptogenic stroke. $\mathrm{N}$ Engl J Med. 2014;370:2467-77.

35. Sanna T, Diener HC, Passman RS, Di Lazzaro V, Bernstein RA, Morillo CA, et al. Cryptogenic stroke and underlying atrial fibrillation. N Engl J Med. 2014;370:2478-86.

36. Broughton ST, O'Neal WT, Salahuddin T, Soliman EZ. The influence of left atrial enlargement on the relationship between atrial fibrillation and stroke. J Stroke Cerebrovasc Dis. 2016;25:1396-402.

37. Hamatani Y, Ogawa H, Takabayashi K, Yamashita Y, Takagi D, Esato $M$, et al. Left atrial enlargement is an independent predictor of stroke and systemic embolism in patients with nonvalvular atrial fibrillation. Sci Rep.;. 2016;6:31042.

38. Chen L, Deng W, Palacios I, Inglessis-Azuaje I, McMullin D, Zhou D, et al. Patent foramen ovale (PFO), stroke and pregnancy. J Investig Med. 2016;64:992-1000.

39. Katsanos AH, Spence JD, Bogiatzi C, Parissis J, Giannopoulos $\mathrm{S}$, Frogoudaki A, et al. Recurrent stroke and patent foramen ovale: A systematic review and meta-analysis. Stroke. 2014;45:3352-9.
40. Khan AR, Bin Abdulhak AA, Sheikh MA, Khan S, Erwin PJ, Tleyjeh I, et al. Device closure of patent foramen ovale versus medical therapy in cryptogenic stroke: A systematic review and metaanalysis. JACC Cardiovasc Interv. 2013;6:1316-23.

41. Eikelboom JW, Quinlan DJ, Hirsh J, Connolly SJ, Weitz JI WA, et al. Laboratory monitoring of non-vitamin $\mathrm{K}$ antagonist oral anticoagulant use in patients with atrial fibrillation. JAMA Cardiol. 2017;18:1609-78.

42. James PA, Oparil S, Carter BL, Cushman WC, DennisonHimmelfarb C, Handler J, et al. Evidence-based guideline for the management of high blood pressure in adults. JAMA. 2014;311:507-20.

43. Piepoli MF, Hoes AW, Agewall S, Albus C, Brotons C, Catapano AL, et al. 2016 European Guidelines on cardiovascular disease prevention in clinical practice. Eur Heart J. 2016;37:2315-81.

44. Niewada M, Kobayashi A, Sandercock PAG, Kamiński B, Członkowska A, International Stroke Trial Collaborative Group. Influence of gender on baseline features and clinical outcomes among 17,370 patients with confirmed ischaemic stroke in the international stroke trial. Neuroepidemiology Karger Publishers. 2005;24:123-8.

45. Appelros $P$, Stegmayr B, Terént A. A review on sex differences in stroke treatment and outcome. Acta Neurol Scand. 2010;121:359-69.

46. Petrea RE, Beiser AS, Seshadri S, Kelly-Hayes M, Kase CS, Wolf PA. Gender differences in stroke incidence and poststroke disability in the framingham heart study. Stroke. 2009;40:1032-7.

47. Goyal M, Menon BK, Van Zwam WH, Dippel DWJ, Mitchell PJ, Demchuk AM, et al. Endovascular thrombectomy after large-vessel ischaemic stroke: A meta-analysis of individual patient data from five randomised trials. Lancet. 2016;387(10029):1723-31.

48. Emberson J, Lees KR, Lyden P, Blackwell L, Albers G, Bluhmki $E$, et al. Effect of treatment delay, age, and stroke severity on the effects of intravenous thrombolysis with alteplase for acute ischaemic stroke: a meta-analysis of individual patient data from randomised trials. Lancet. 2014;384:1929-35.

49. Saver JL, Fonarow GC, Smith EE, Reeves MJ, Grau-Sepulveda MV, Pan W, et al. Time to treatment with intravenous tissue plasminogen activator and outcome from acute ischemic stroke. JAMA. 2013;309:2480-8.

50. Saver JL, Filip B, Hamilton S, Yanes A, Craig S, Cho M, et al. Improving the reliability of stroke disability grading in clinical trials and clinical practice: the Rankin Focused Assessment (RFA). Stroke. 2010;41:992-5.

51. Kent DM, Price LL, Ringleb P, Hill MD, Selker HP. Sex-based differences in response to recombinant tissue plasminogen activator in acute ischemic stroke: A pooled analysis of randomized clinical trials. Stroke. 2005;36:62-5.

52. Johnbull EA, Lau BD, Schneider EB, Streiff MB. Rate vs Percentage of Patients Who Received SCIP VTE-2 Uncertainty in Management of Carotid Stenosis in Women. To the Editor A review of the "management of carotid stenosis". 2014;149:401-3.

53. Pancholy SB, Sharma PS, Pancholy DS, Patel TM, Callans DJ, Marchlinski FE. Meta-analysis of gender differences in residual stroke risk and major bleeding in patients with nonvalvular atrial fibrillation treated with oral anticoagulants. Am J Cardiol. 2014;113:485-90.

54. Christensen H, Bentsen L, Christensen L. Update on specificities of stroke in women. Presse Med. Elsevier Masson SAS; 2016;45:e409-18. 